University of Nebraska - Lincoln

DigitalCommons@University of Nebraska - Lincoln

Robert Streubel Papers

Research Papers in Physics and Astronomy

6-26-2017

\title{
Vortex circulation patterns in planar microdisk arrays
}

Sven Velten

Lawrence Berkeley National Laboratory, svelten@physnet.uni-hamburg.de

Robert Streubel

Lawrence Berkeley National Laboratory, streubel@unl.edu

Alan Farhan

Lawrence Berkeley National Laboratory

Noah Kent

Lawrence Berkeley National Laboratory

Mi Young Im

Lawrence Berkeley National Laboratory

See next page for additional authors

Follow this and additional works at: https://digitalcommons.unl.edu/physicsstreubel

Part of the Atomic, Molecular and Optical Physics Commons, Condensed Matter Physics Commons, and the Other Physics Commons

Velten, Sven; Streubel, Robert; Farhan, Alan; Kent, Noah; Im, Mi Young; Scholl, Andreas; Dhuey, Scott; Behncke, Carolin; Meier, Guido; and Fischer, Peter, "Vortex circulation patterns in planar microdisk arrays" (2017). Robert Streubel Papers. 15.

https://digitalcommons.unl.edu/physicsstreubel/15

This Article is brought to you for free and open access by the Research Papers in Physics and Astronomy at DigitalCommons@University of Nebraska - Lincoln. It has been accepted for inclusion in Robert Streubel Papers by an authorized administrator of DigitalCommons@University of Nebraska - Lincoln. 


\section{Authors}

Sven Velten, Robert Streubel, Alan Farhan, Noah Kent, Mi Young Im, Andreas Scholl, Scott Dhuey, Carolin Behncke, Guido Meier, and Peter Fischer 


\section{Vortex circulation patterns in planar microdisk arrays}

Cite as: Appl. Phys. Lett. 110, 262406 (2017); https://doi.org/10.1063/1.4990990

Submitted: 25 April 2017 • Accepted: 18 June 2017 • Published Online: 30 June 2017

Sven Velten, Robert Streubel, (D) Alan Farhan, et al.

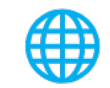

\section{ARTICLES YOU MAY BE INTERESTED IN}

The design and verification of MuMax3

AIP Advances 4, 107133 (2014); https://doi.org/10.1063/1.4899186

Magnetic vortex nucleation modes in static magnetic fields

AIP Advances 7, 105103 (2017); https://doi.org/10.1063/1.5006235

Launching a new dimension with 3D magnetic nanostructures

APL Materials 8, 010701 (2020); https://doi.org/10.1063/1.5134474

\section{Challenge us.}

What are your needs for periodic signal detection?

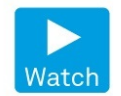

Zurich

Instruments

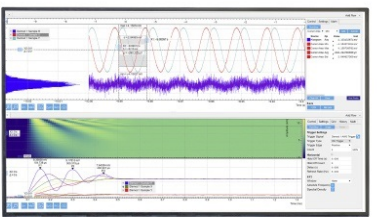

- 


\title{
Vortex circulation patterns in planar microdisk arrays
}

\author{
Sven Velten, ${ }^{1,2, a)}$ Robert Streubel, ${ }^{1, b)}$ Alan Farhan, ${ }^{3}$ Noah Kent, ${ }^{1,4}$ Mi-Young Im, ${ }^{5,6}$ \\ Andreas Scholl, ${ }^{3}$ Scott Dhuey, ${ }^{7}$ Carolin Behncke, ${ }^{2}$ Guido Meier, ${ }^{8}$ and Peter Fischer ${ }^{1,4}$ \\ ${ }^{1}$ Materials Sciences Division, Lawrence Berkeley National Laboratory, 1 Cyclotron Road, Berkeley, \\ California 94720, USA \\ ${ }^{2}$ Institut für Nanostruktur- und Festkörperphysik, Universität Hamburg, Jungiusstrasse 11, \\ 20355 Hamburg, Germany \\ ${ }^{3}$ Advanced Light Source, Lawrence Berkeley National Laboratory, 1 Cyclotron Road, Berkeley, \\ California 94720, USA \\ ${ }^{4}$ Physics Department, University of California, 1156 High Street, Santa Cruz, California 95064, USA \\ ${ }^{5}$ Center for X-ray Optics, Lawrence Berkeley National Laboratory, Berkeley, California 94720, USA \\ ${ }^{6}$ Department of Emerging Materials Science, Daegu Gyeongbuk Institute of Science and Technology, \\ Daegu 711-873, South Korea \\ ${ }^{7}$ Molecular Foundry, Lawrence Berkeley National Laboratory, 1 Cyclotron Road, Berkeley, \\ California 94720, USA \\ ${ }^{8}$ Max-Planck Institute for the Structure and Dynamics of Matter, Luruper Chaussee 149, \\ 22761 Hamburg, Germany
}

(Received 25 April 2017; accepted 18 June 2017; published online 30 June 2017)

\begin{abstract}
We report a magnetic X-ray microscopy study of the pattern formation of circulation in arrays of magnetic vortices ordered in a hexagonal and a honeycomb lattice. In the honeycomb lattice, we observe at remanence an ordered phase of alternating circulations, whereas in the hexagonal lattice, small regions of alternating lines form. A variation in the edge-to-edge distance shows that the size of those regions scales with the magnetostatic interaction. Micromagnetic simulations reveal that the patterns result from the formation of flux closure states during the nucleation process. Published by AIP Publishing. [http://dx.doi.org/10.1063/1.4990990]
\end{abstract}

The coupling of magnetic nanostructures in lateral arrangements leads to a collective behavior of spin textures, which results, for example, in exotic phase transitions, such as the spin ice phase, ${ }^{1}$ or specific spectra for spin excitations in so-called magnonic crystals. ${ }^{2,3}$ Recently, chiral spin textures have stepped into the spotlight due to their possible impact on spintronic devices. ${ }^{4-7}$ These spin textures can be formed as a result of confinement in magnetic structures. Examples are magnetic vortices that are formed in circular microdisks. Such a vortex has a circulating in-plane texture (circulation) that can rotate clockwise or counter-clockwise, and a vortex core at its center (polarization) can point up or down. The orientations of both circulation (clockwise/counter-clockwise) and polarization (up/down) are degenerated in isolated disks. However, when arranged in arrays, magnetic vortices are coupled due to magnetostatic interactions arising if an external magnetic field is applied. This leads to a collective ordering of the polarization states, if the magnetic field is oscillating with specific frequencies. ${ }^{8}{ }^{89}$ Moreover, the vortex core excitation spectrum reveals band-structure like behavior. ${ }^{10,11}$ For circulation states, patterns are reported after applying a strong magnetic field pulse to disk pairs ${ }^{12}$ or to arrays of curved thin film structures. ${ }^{13,14}$ The formation of those patterns is affected by the coupling strength and thermal excitations. However, a profound study of the vortex circulation pattern as a function of the interplay between magnetostatics and thermal fluctuations is still lacking.

Here, we report an investigation of arrays of planar disks ordered in hexagonal and honeycomb lattices. We study the

\footnotetext{
${ }^{a}$ E-mail: svelten@physnet.uni-hamburg.de

b)E-mail: streubel@lbl.gov
}

formation of the patterns in the circulation of the magnetic vortices by high resolution magnetic soft X-ray microscopy. By systematically varying the lattice constant and thus the strengths of the magnetostatic interaction between neighboring disks, we observe a clear impact on the pattern formation and the pattern size. Our findings prove that the pattern formation takes place on a completely different energy scale than the alternations of vortex annihilation and nucleation fields in vortex arrays.

The microdisk arrays were patterned by utilizing electron beam lithography and ultrasonic agitation development at $5^{\circ} \mathrm{C}$ to promote the removal of degraded molecules ${ }^{15}$ and to provide the required spatial resolution for fabricating well defined microstructures [surface roughness: $(3.8 \pm 1.8) \mathrm{nm}$; deviation from an ideal round disk: $\left.(0.005 \pm 0.003) \mu \mathrm{m}^{2}\right]$. Disks with a diameter of $1 \mu \mathrm{m}$ and an edge-to-edge spacing $\mathrm{d}_{e}$ ranging from $200 \mathrm{~nm}$ down to $70 \mathrm{~nm}$ were arranged into areas of $25 \mu \mathrm{m} \times 25 \mu \mathrm{m}$ with hexagonal or honeycomb symmetry. Electron beam evaporation of permalloy (Py, $\mathrm{Ni}_{80} \mathrm{Fe}_{20}$ ) followed by lift off led to $40 \mathrm{~nm}$ thin soft-magnetic disks of the desired geometry. Images of the structures with their symmetry directions [10] and [01] are shown in Figs. 1(a) and 1(b). In those Py disks, the magnetic vortex ground state is favored. The circulations of the vortices arrange in certain patterns throughout the disk array, as illustrated in Figs. 1(c) and 1(d).

The interaction responsible for the pattern formation is of pure magnetostatic nature, ${ }^{16}$ whereas the remanent vortex state is virtually stray field free; small lateral displacements of the vortex core induced by an external magnetic field cause surface charges at the disk edges $[\sigma(\vec{r})=\vec{M}(\vec{r}) \cdot \vec{n}(\vec{r})$, with local magnetization $\vec{M}(\vec{r})$ and surface normal $\vec{n}(\vec{r})$ ] being large in the beginning of the vortex nucleation process when 

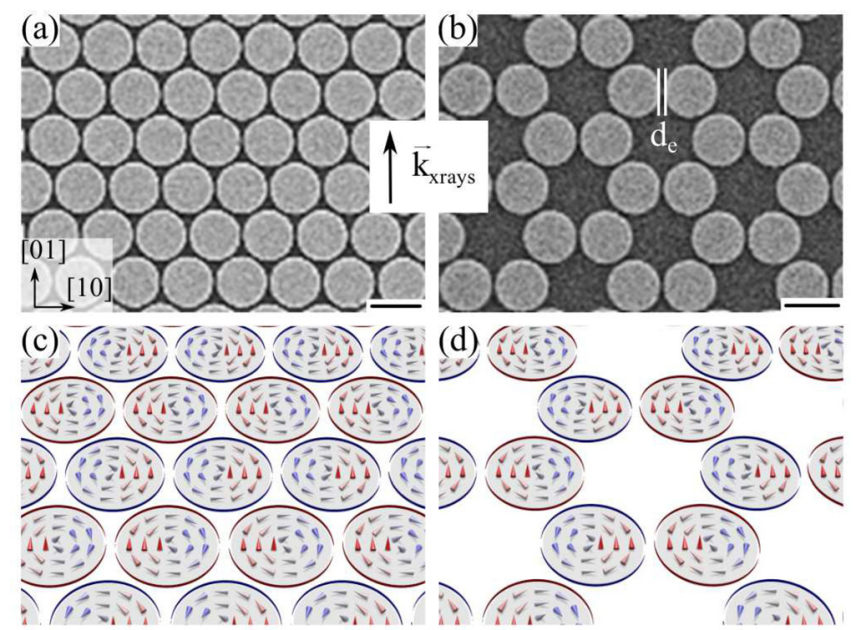

(e)

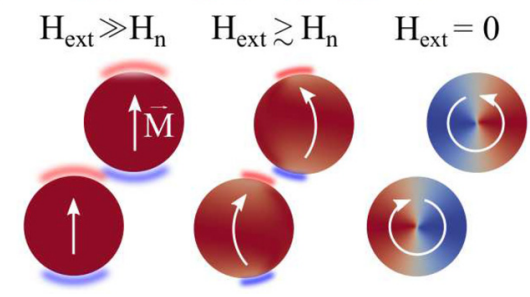

FIG. 1. Scanning electron micrographs of the vortex lattices with the edgeto-edge distance $\mathrm{d}_{e}=70 \mathrm{~nm}$ for the (a) hexagonal lattice and (b) honeycomb lattice. The illustrated symmetry directions [10] and [01] are valid for both lattices. The mid-inset illustrates the X-ray wavevector component $\vec{k}_{\text {xrays }}$ lying in the sample plane. An external magnetic field $\overrightarrow{\mathrm{H}}_{\mathrm{sat}}$ is applicable, oriented along the directions [10] or [01]. Scale bars indicate $1 \mu \mathrm{m}$. Schematics of the magnetic ground state at remanence in the (c) hexagonal and (d) honeycomb disk arrays. In both cases, the circulation states (either clockwise or counter-clockwise) of the vortices form patterns. (e) Micromagnetic simulation of a diagonal vortex pair with a decreasing external field $\mathrm{H}_{\text {ext }}$ from a magnitude much bigger than the nucleation field $\mathrm{H}_{n}$ (left picture) to remanence (right). Also shown is the nucleation state (middle). The local magnetization is illustrated by a white arrow and the colorized disk with a red (parallel to $\vec{k}$, as indicated in the mid-inset above) to blue (antiparallel) contrast. Positive and negative magnetic surface charges are highlighted by red and blue shadows, respectively.

applying in-plane magnetic fields larger than the annihilation/ nucleation fields. In individual and perfectly isolated magnetic vortex structures, the selection of polarization and circulation is completely random. This degeneracy is lifted in arrangements of magnetostatically interacting vortices, e.g., in pairs. Here, the circulation is predetermined by the orientation of the in-plane magnetic field relative to the pair. ${ }^{12}$ The polarization state does not affect the interaction since the vortex core radius is much smaller than the disk radius. ${ }^{12}$ An exemplary simulation of the nucleation process of diagonally aligned vortices is shown in Fig. 1(e). The micromagnetic simulations are performed by using Nmag v.0.2, ${ }^{17}$ the Hlib library, ${ }^{18,19}$ and typical material parameters for Py: ${ }^{20}$ saturation magnetization $M_{s}=860 \mathrm{kA} / \mathrm{m}$ and exchange stiffness $A=1.3 \times 10^{-11} \mathrm{~J} / \mathrm{m}$. The interaction between surface charges in the saturated state (left picture) results in a flux closure state, when the external field is lowered to the regime of the nucleation field (middle). At remanence, the circulations are alternatingly ordered (right). A change in the orientation of the external field varies the distance between the surface charges and thus the interaction strength.

This scheme provides means to tailor the vortex circulation pattern throughout an entire array with certain structural symmetry. Figure 2 depicts the vortex circulation patterns at remanence after saturating along the [01] [Figs. 2(a) and 2(c)] and [10] [Figs. 2(b) and 2(d)] directions. The results are obtained by utilizing magnetic transmission $\mathrm{X}$-ray microscopy (MTXM) and X-ray photoemission electron microscopy (X-PEEM), both located at the Advanced Light Source (ALS) in Berkeley, CA. By tuning the circularly polarized X-rays to the $\mathrm{Fe} L_{3}$-edge $(706.8 \mathrm{eV})$, a magnetic contrast is measured by $\mathrm{X}$-ray magnetic circular dichroism (XMCD). The effect provides an absorption dependency of the relative orientation of the incident photon helicity and the local magnetization. The wavevector component $\vec{k}_{\text {xrays }}$ of the photons in the sample plane is depicted in Fig. 1. Dark areas in the XMCD pictures refer to a magnetization parallel to $\vec{k}_{\text {xrays }}$, whereas an antiparallel alignment is attributed to bright areas.

The honeycomb array previously saturated along [01] [Fig. 2(a)] shows an extended alternating circulation pattern as expected beforehand [Fig. 1(d)] due to flux closure states throughout the lattice during the nucleation [middle picture in Fig. 1(e)]. A saturation along [10], however, results in a disappearance of the pattern [Fig. 2(b)]. The lack of diagonal neighbors along the flux closure chains destabilizes the ordering. Additionally, a disk is subjected to competing interactions to form the flux closure state with its two diagonal neighbors (aligned perpendicularly to the saturation direction). This leads to the phenomenon of a frustrated circulation state, which was first reported in hemispherical cap structures. ${ }^{13}$ As a consequence, the result is a disordered circulation pattern.

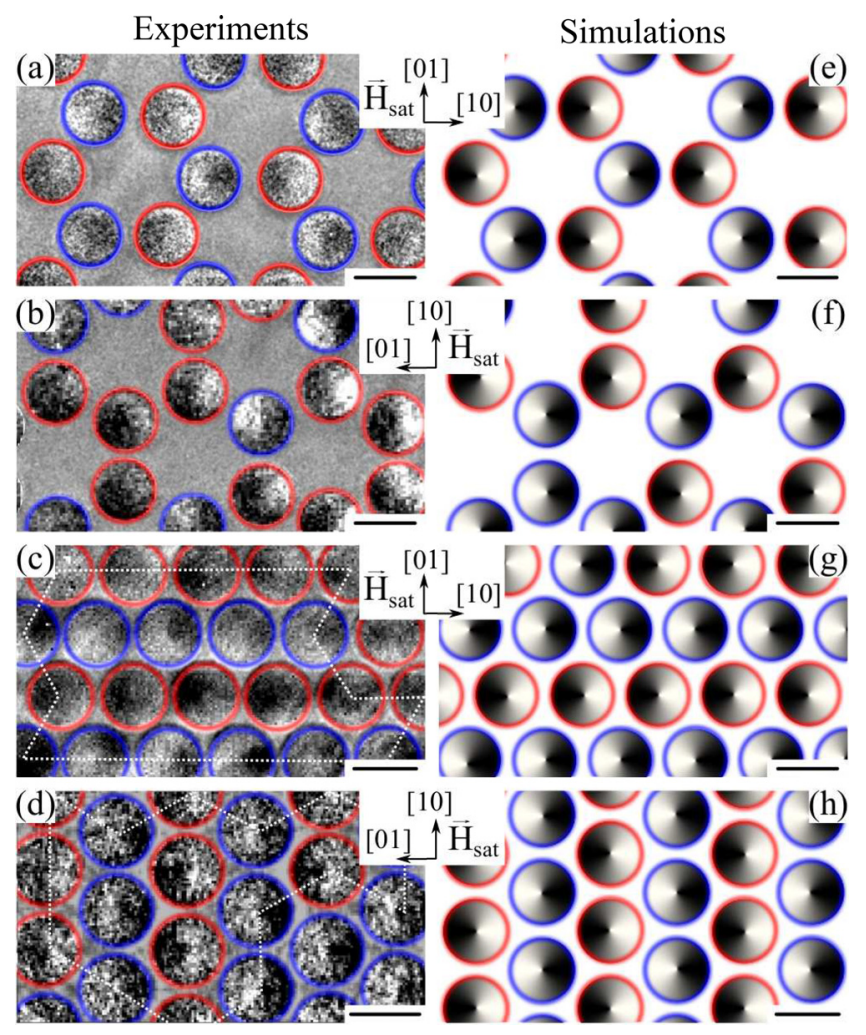

FIG. 2. MTXM results for a (a) and (b) honeycomb and (c) and (d) hexagonal vortex lattice. The disks are previously saturated along [01] in (a) and (c) and [10] in (b) and (d). The disks are highlighted in red or blue to indicate the clockwise or counter-clockwise vortex circulation. (e)-(h) Corresponding micromagnetic simulation results. Scale bars indicate $1 \mu \mathrm{m}$. 
The hexagonal array shows a completely different behavior. For both saturation directions, the circulation states in the hexagonal lattice form an alternating line pattern [Figs. 2(c) and 2(d)]. They are a consequence of the combination of stray field minimization and circulation frustration. The flux closure states during the nucleation are built throughout the lattice, which generates the alternating line pattern. This competes with the tendency to disorder due to the existence of competing interactions of the diagonal neighbors. Hence, the ordering can only occur around lattice defects and imperfect disks, where a slightly higher vortex nucleation field of a disk leads to a symmetry breaking. The pattern size should therefore be influenced by lattice defects, magnetostatic interaction strength and thermal fluctuations. Besides that, thermal fluctuations are expected to play a major role in the lateral extent since the energy difference between both circulation states is small. ${ }^{14,21}$

Figures 2(e)-2(h) show the corresponding states obtained from micromagnetic simulations using the same field protocol as in the MTXM measurements. The results are in very good agreement with the experiments. Since lattice defects are lacking in the simulations, the patterns are expected to be suppressed in the hexagonal lattice, as they have no nucleation center to form around. Despite that, the alternating patterns are still formed in our simulations [Figs. 2(g) and 2(h)]. The reasons are boundary effects in the simulated 6 disk times 6 disk array. Vortices at the lattice boundaries have a higher nucleation field because of the weaker magnetostatic interaction. ${ }^{22}$ A subsequent simulation with periodic boundary conditions resulted in random circulation states.

Note that the lack of a proper mechanism to mimic temperature in our simulations, and therefore not accounting for thermal fluctuations, resulted in significantly larger vortex circulation patterns than experimentally observed at room temperature.

The pattern size is a parameter to measure the influence of lattice defects, thermal fluctuations, and magnetostatic coupling strength on circulation patterns. The coupling strength monotonically decreases with increasing edge-to-edge distance $\mathrm{d}_{e}{ }^{22}$ Thus, for the hexagonal lattice, Fig. 3(a) shows a decreasing number of disks $\mathrm{N}_{D}$ forming a pattern region with increasing lattice spacing. The size depends strongly on the interaction strengths to overcome thermal agitations. The experiments performed at room temperature show a threshold edge-to-edge distance of below $80 \mathrm{~nm}$ to order circulation states. The threshold is well below the expected distance, when just considering the magnetostatic coupling, which leads to a significant decrease in nucleation and annihilation fields in vortex arrays, if $\mathrm{d}_{e}$ is already lowered to half the disk radius $\left(250 \mathrm{~nm}\right.$ in our case). ${ }^{22}$ This points out the impact of thermal fluctuations, resulting in a higher coupling energy needed to form the patterns. The distribution of lattice defects is random and independent of $\mathrm{d}_{e}$ and therefore cannot explain the low threshold.

A more quantitative non-local analysis of the magnetostatic coupling can be obtained by calculating correlations, which are often used to describe ordering phenomena in a two-state system, e.g., in artificial spin ice. ${ }^{1,23}$ For that purpose, X-PEEM measurements were performed providing us a large view on the lattice, which increases the statistics
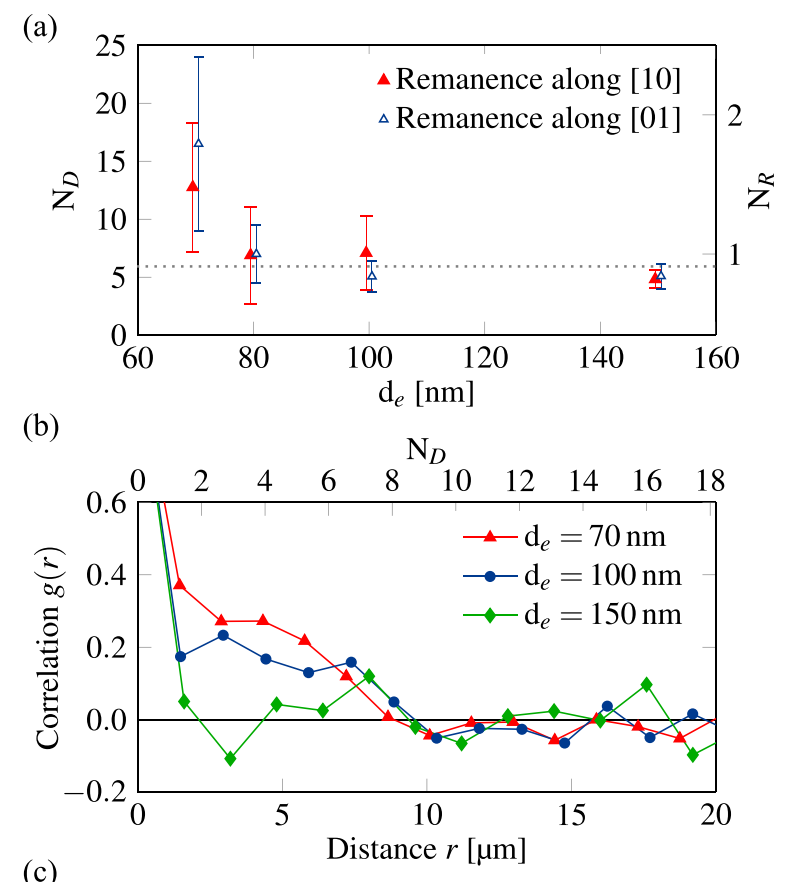

(c)

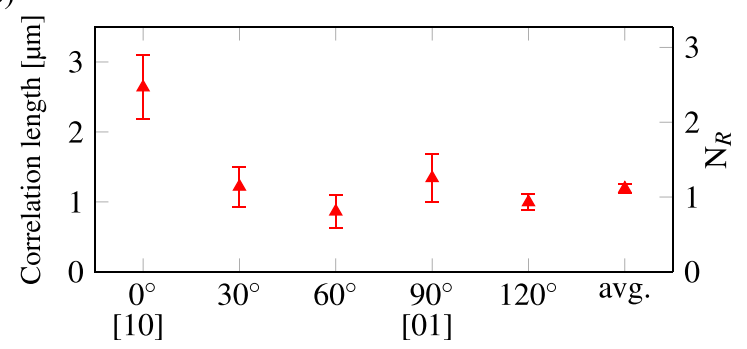

FIG. 3. (a) Average number of disks $\mathrm{N}_{D}$ in the alternating line regions in the hexagonal lattice as a function of the edge-to-edge distance $\mathrm{d}_{e}$ obtained by MTXM measurements. The gray dotted line illustrates a random distribution of circulation states (disks are not interacting). (b) Spatial correlation along [10], when the lattice was saturated along [01], as a function of the distance and the number of disks $\mathrm{N}_{D}$ along the axis for different $\mathrm{d}_{e}$ values. Calculated from X-PEEM measurements. (c) Derived correlation lengths along different major axes $\left(0^{\circ}=[10]\right.$ and $\left.90^{\circ}=[01]\right)$ and minor axes $\left(30^{\circ}, 60^{\circ}\right.$, and $120^{\circ}$ ) and the spatial average for the lattice with $\mathrm{d}_{e}=70 \mathrm{~nm}$, which was saturated along [01]. The spatial average corresponds to a measurement of the average radius of the circulation patterns, expressed as the number of disks $\mathrm{N}_{R}$. For comparison, this quantity is added as an axis in (a).

significantly (over 400 disks). The resulting XMCD pictures (not shown) are analyzed by calculating the spatial correlation $g(r)$ which is defined by ${ }^{24}$

$$
g(r)=\left\langle c_{i} c_{j}\right\rangle-\left\langle c_{i}\right\rangle\left\langle c_{j}\right\rangle .
$$

Here, $r$ denotes the distance between the disks $i$ and $j$ with vortex circulation $c_{i}( \pm 1)$ and $c_{j}( \pm 1) .\langle\ldots\rangle$ represents a spatial average over all disk pairs separated by the same distance $r$. The sign of the correlation refers to a homocircular (positive value) or an alternating (negative) coupling. Figure 3(b) plots the correlation $g(r)$ as a function of the distance for different edge-to-edge distances $\mathrm{d}_{e}$ calculated from the $\mathrm{X}$-PEEM measurements along the [10] axis. It gives us access to the length of the homocircular disk chains along [10] (upper axis in the plot). The vanishing correlation for large interdistances $\left(\mathrm{d}_{e}=150 \mathrm{~nm}\right)$ refers to the regime, where the formation of circulation patterns is unfavorable due to the decreasing magnetostatic interaction and the contributions of 
thermal fluctuations. Note that the oscillatory behavior of the correlation function within the first mircometers is assigned to statistical errors rather than of the physical origin since it also occurs at large distances, where the correlation has to be zero. However, for $\mathrm{d}_{e}=100 \mathrm{~nm}$ and particularly for $\mathrm{d}_{e}=70 \mathrm{~nm}$, the disks are positively coupled, corresponding to a homocircular configuration along [10], which is in agreement with the MTXM results. For measuring the correlation strength, a correlation length is defined as the distance, where $g(r)$ is decreased to $1 / e$. Due to the expected alternating character of the correlation function along certain axes, the length was calculated for $|g(r)|$. The correlation length along different axes and the spatial average are plotted in Fig. 3(c) for the lattice with $\mathrm{d}_{e}=70 \mathrm{~nm}$, initially saturated along [01]. Along all axes, the disks are coupled and most prominently along [10], which shows the homocircular chains. The spatial average as a measurement of the average radius of the circulation patterns is about $1 \mu \mathrm{m}$, corresponding to one disk in every direction, as expressed in the quantity $\mathrm{N}_{R}$ in the figure. To compare it with the MTXM results, $\mathrm{N}_{R}$ as a function of $\mathrm{d}_{e}$ is shown in Fig. 3(a), assuming an isotropic pattern formation. The resulting average radius seems to be bigger because the isotropic assumption is not accurate. The X-PEEM measurements show that the patterns are formed but are not homogeneous along all axes [Fig. 3(c)]. They are stretched along [10], resulting in a smaller average pattern radius.

In conclusion, we have observed by X-ray imaging experiments that circulation patterns are formed on a different energy scale than the scale of annihilation and nucleation field variations in vortex arrays. Micromagnetic simulations point to the reason which is the interplay between the formation of flux-closure states during vortex nucleation and emerging circulation frustrations, where one favors order and the other prefers disorder. The selection between the two regimes is primarily determined by the coupling strength and thermal fluctuations. It shows that the circulation state can still be degenerated (above $\mathrm{d}_{e}=100 \mathrm{~nm}$ ) even though the annihilation and nucleation fields are strongly decreased by the vortex-vortex interaction. Our result can support the design of vortex data storage devices based on degenerated vortices and field sensor applications. 4,25,26 $^{-2}$

We would like to thank Ulrich Merkt for fruitful discussions and his support. This work was supported by the SFB668 of the German Science Foundation and the Cluster of Excellence "The Hamburg Center for Ultrafast Imaging (CUI)." The X-ray microscopy work at the ALS was funded by the U.S. Department of Energy, Office of Science, Basic Energy Sciences under Contract No. DE-AC02-05-CH11231. R.S., N.K., and P.F. acknowledge support from the U.S. Department of Energy, Office of Science, Basic Energy Sciences, Materials Sciences and Engineering Division under Contract No. DE-AC02-05-CH11231 (NEMM program
MSMAG). M.Y.I. acknowledges support from Leading Foreign Research Institute Recruitment Program through the NRF of Korea funded by the MEST (2012K1A4A3053565) and from the DGIST R\&D program of the Ministry of Science, ICT and future Planning (17-BT-02). A.F. acknowledges support from the Swiss national science foundation.

${ }^{1}$ R. Wang, C. Nisoli, R. Freitas, J. Li, W. McConville, B. Cooley, M. Lund, N. Samarth, C. Leighton, V. Crespi, and P. Schiffer, Nature 439, 303 (2006).

${ }^{2}$ V. Kruglyak and A. Kuchko, Phys. B: Condens. Matter 339, 130 (2003).

${ }^{3}$ H. Puszkarski and M. Krawczyk, Interfacial Effects and Novel Properties of Nanomaterials, Solid State Phenomena (Trans Tech Publications, 2003), Vol. 94, pp. 125-134.

${ }^{4}$ B. Van Waeyenberge, A. Puzic, H. Stoll, K. W. Chou, T. Tyliszczak, R. Hertel, M. Fähnle, H. Brückl, K. Rott, G. Reiss, I. Neudecker, D. Weiss, C. H. Back, and G. Schütz, Nature 444, 461 (2006).

${ }^{5}$ A. Fert, V. Cros, and J. Sampaio, Nat. Nanotechnol. 8, 152 (2013).

${ }^{6}$ R. Wiesendanger, Nat. Rev. Mater. 1, 16044 (2016).

${ }^{7}$ A. Soumyanarayanan, N. Reyren, A. Fert, and C. Panagopoulos, Nature 539, 509 (2016).

${ }^{8}$ S. Jain, V. Novosad, F. Fradin, J. Pearson, V. Tiberkevich, A. Slavin, and S. Bader, Nat. Commun. 3, 1330 (2012).

${ }^{9}$ C. F. Adolff, M. Hänze, A. Vogel, M. Weigand, M. Martens, and G. Meier, Phys. Rev. B 88, 224425 (2013).

${ }^{10}$ C. F. Adolff, M. Hänze, M. Pues, M. Weigand, and G. Meier, Phys. Rev. B 92, 024426 (2015).

${ }^{11}$ C. Behncke, M. Hänze, C. F. Adolff, M. Weigand, and G. Meier, Phys. Rev. B 91, 224417 (2015).

${ }^{12}$ S. Jain, Y. Ren, A. O. Adeyeye, and N. Singh, Phys. Rev. B 80, 132401 (2009).

${ }^{13}$ R. Streubel, D. Makarov, F. Kronast, V. Kravchuk, M. Albrecht, and O. G. Schmidt, Phys. Rev. B 85, 174429 (2012).

${ }^{14}$ R. Streubel, F. Kronast, C. F. Reiche, T. Mühl, A. U. B. Wolter, O. G. Schmidt, and D. Makarov, Appl. Phys. Lett. 108, 042407 (2016).

${ }^{15}$ T. Miyata, Y. Minamino, S. Ida, and T. Minami, J. Vac. Sci. Technol. A 22, 1711 (2004)

${ }^{16}$ R. Streubel, P. Fischer, F. Kronast, V. P. Kravchuk, D. D. Sheka, Y. Gaididei, O. G. Schmidt, and D. Makarov, J. Phys. D: Appl. Phys. 49, 363001 (2016).

${ }^{17}$ T. Fischbacher, M. Franchin, G. Bordignon, and H. Fangohr, IEEE Trans. Mag. 43, 2896 (2007).

${ }^{18} \mathrm{~W}$. Hackbusch, Hierarchische Matrizen: Algorithmen Und Analysis (Springer Science \& Business Media, 2009).

${ }^{19}$ S. Börm, Efficient Numerical Methods for Non-Local Operators: H2Matrix Compression, Algorithms and Analysis (European Mathematical Society, 2010), Vol. 14.

${ }^{20}$ R. Streubel, F. Kronast, U. K. Rößler, O. G. Schmidt, and D. Makarov, Phys. Rev. B 92, 104431 (2015).

${ }^{21}$ M.-Y. Im, K.-S. Lee, A. Vogel, J.-I. Hong, G. Meier, and P. Fischer, Nat. Commun. 5, 5620 (2014).

${ }^{22}$ K. Y. Guslienko, V. Novosad, Y. Otani, H. Shima, and K. Fukamichi, Phys. Rev. B 65, 024414 (2001).

${ }^{23}$ S. Zhang, I. Gilbert, C. Nisoli, G.-W. Chern, M. J. Erickson, L. O’Brien, C. Leighton, P. E. Lammert, V. H. Crespi, and P. Schiffer, Nature 500, 553 (2013).

${ }^{24}$ W. Nolting, "Phasenübergänge," in Grundkurs Theoretische Physik 6: Statistische Physik (Springer, Berlin, Heidelberg, 2014), pp. 279-415.

${ }^{25}$ S. Bohlens, B. Krüger, A. Drews, M. Bolte, G. Meier, and D. Pfannkuche, Appl. Phys. Lett. 93, 142508 (2008).

${ }^{26}$ J. Zimmer, A. Satz, W. Raberg, H. Brueckl, and D. Suess, "Device, magnetic sensor device and method," U.S. patent 20150185297 A1 (2013). 